\title{
La loi sur l'économie sociale et solidaire : un regard juridique bienveillant
}

The law on the social and solidarity economy: A supportive legal perspective

\section{La ley de economía social y solidaria: una mirada jurídica benévolal}

\section{David Hiez}

Numéro 334, octobre 2014

La loi ESS : historique

Social and Solidarity: Economy History and Legislation

URI : https://id.erudit.org/iderudit/1027275ar

DOI : https://doi.org/10.7202/1027275ar

Aller au sommaire du numéro

Éditeur(s)

Association Recma

ISSN

1626-1682 (imprimé)

2261-2599 (numérique)

Découvrir la revue

Citer cet article

Hiez, D. (2014). La loi sur l'économie sociale et solidaire : un regard juridique bienveillant. Revue internationale de l'économie sociale, (334), 44-56.

https://doi.org/10.7202/1027275ar
Résumé de l'article

La loi sur l'économie sociale et solidaire votée en juillet dernier est présentée comme une grande loi, à la fois rénovatrice et structurante de l'ESS. Les commentaires qu'elle suscite sont, si l'on excepte les officiels, prudents ou critiques. Ces hésitations sont à la hauteur des attentes qu'elle suscite et que le juriste juge parfois démesurées. La loi n’apparaît en effet pas

fondamentalement différente de ses émules espagnole et québécoise, du moins dans sa fonction structurante. Sur le plan strictement national, elle tranche avec la précédente, de 1992, qui avait une même ampleur. Outre l'influence des débats suscités par l'économie solidaire, voire l'entreprise sociale, elle se caractérise par la baisse de l'attraction pour les mécanismes capitalistes et la réaffirmation ou le développement des principes plus spécifiques à l'ESS. 


\title{
LA LOI SUR L'ÉCONOMIE SOCIALE ET SOLIDAIRE : UN REGARD JURIDIQUE BIENVEILLANT
}

\author{
par David Hiez*
}

\author{
* Professeur à l'université \\ du Luxembourg. Mél.: david. \\ hiez@uni.lu.
}

La loi sur l'économie sociale et solidaire votée en juillet dernier est présentée comme une grande loi, à la fois rénovatrice et structurante de l'ESS. Les commentaires qu'elle suscite sont, si l'on excepte les officiels, prudents ou critiques. Ces hésitations sont à la hauteur des attentes qu'elle suscite et que le juriste juge parfois démesurées. La loi n'apparaît en effet pas fondamentalement différente de ses émules espagnole et québécoise, du moins dans sa fonction structurante. Sur le plan strictement national, elle tranche avec la précédente, de 1992, qui avait une même ampleur. Outre l'influence des débats suscités par l'économie solidaire, voire l'entreprise sociale, elle se caractérise par la baisse de l'attraction pour les mécanismes capitalistes et la réaffirmation ou le développement des principes plus spécifiques à l’ESS.

\section{The law on the social and solidarity economy: A supportive legal perspective}

The law passed last July was presented as a major piece of legislation that would reinvigorate and restructure the social and solidarity economy. Apart from official statements, reactions to the law have been guarded or critical. These reservations mirror the expectations that the law generated, expectations that a lawyer might consider unrealistic. The law does not appear to be fundamentally different from its counterparts in Spain and Quebec at least in its organisational aspects. In the French context, however, the law is a departure from the previous law in 1992, which had comparable ambitions. Besides the influence of the issues raised by the solidarity economy and social enterprises, the law features fewer attractions for capitalist mechanisms and reaffirms and strengthens principles that are more specific to the social and solidarity economy.

\section{La ley de economía social y solidaria : una mirada jurídica benévolal}

La ley votada en julio pasado ha sido presentada como una grande ley, a la vez renovadora y estructurante para la economía solidaria. Los comentarios que suscita son, aparte de los oficiales, prudentes o críticos. Tales vacilaciones están a la altura de las expectativas que la ley ha suscitado y a veces estas son consideradas excesivas por el jurista. En efecto, la ley parece no fundamentalmente diferente de sus equivalentes española y de Québec, al menos en su función estructurante. Al nivel puramente nacional, la ley contrasta con la precedente que tenia la misma importancia en 1992. Además de influir en los debates sobre la economía solidaria, incluido la empresa social, se caracteriza por el descenso de atracción para los mecanismos capitalistas y por la reafirmación o el desarrollo de los principios más específicos de la economía solidaria. 
Le 21 juillet dernier, le Parlement français a définitivement adopté le projet de loi sur l'économie sociale et solidaire, sans opposition ni saisine ultérieure du Conseil constitutionnel. Celui-ci a donc pu être promulgué rapidement et a été publié au Journal officiel le $1^{\mathrm{er}}$ août 2014. Faute de dispositions transitoires générales, la loi est entrée en vigueur le 2 août. Composée d'une centaine d'articles et, surtout, consistant dans environ quatre-vingts pages, elle est remarquable par son étendue. Elle tranche en cela avec ses émules

(1) Ley 5-2011 de 29 de marzo de economía social, Boletín oficial del Estado, mercredi 30 mars $2011, \mathrm{n}^{\circ} 76$, sec. I., p. 33023.

(2) Loi sur l'économie sociale, Recueil annuel des lois du Québec, 2013, chapitre XXII. (3) On sait que les vocables varient selon les pays sans que cela recouvre toujours des différences substantielles comparables. La loi québécoise par exemple porte sur l'«économiesociale», mais vise dans son quatrième considérant l'«économie solidaire et durable». Ce n'est certainement pas une allusion à la terminologie hexagonale, dont les débats parfois picrocholins nesesont pas exportés. (4) Ces organismes sont nommément désignés dans la loi québécoise (art. 5), tandis que la loi espagnole définit les conditions de leur représentativité (art. 7). en Espagne ${ }^{(1)}$ ou au Québec ${ }^{(2)}$, dont les deux lois sont beaucoup plus courtes (une dizaine d'articles tenant sur moins de cinq pages) et ont un contenu voisin: une définition de l'économie sociale ${ }^{(3)}$, l'insertion de l'économie sociale dans les politiques publiques, la détermination des organismes partenaires pour l'élaboration de ces dernières ${ }^{(4)}$.

Par contraste, la loi française peut sembler de prime abord beaucoup plus ambitieuse. Elle contient également en effet une définition de l'économie sociale et solidaire et la détermination d'un cadre d'élaboration des politiques publiques et d'expression officielle du secteur. Cette base est cependant complétée par de nombreuses dispositions concernant toutes les branches de l'ESS. Cette profusion peut pourtant être analysée comme affaiblissant l'ensemble par un effet de dilution. Par ailleurs, la loi n'institue pas d'imbrication générale dans les politiques publiques, comme l'ont fait les émules étrangères. Quoi qu'il en soit, elle s'inscrit dans un contexte international d'intérêt croissant pour les entreprises du secteur: même l'Union européenne, dont on se plaît à relever le désengagement depuis la fin de la prédominance de l'Europe latine sur la question, multiplie les initiatives ces dernières années. Si l'on situe à présent cette loi dans le contexte national, elle matérialise à la fois le retour du soutien des pouvoirs publics, fût-il éphémère ou inégal, et la confirmation de la fin de l'attraction du droit des sociétés capitalistes dans les réformes des organisations du secteur. Cette loi est en effet l'initiative d'un gouvernement de gauche, inscrite dans son programme électoral et portée par un ministre volontariste (Benoît Hamon). Si les évolutions récentes ont manifesté un recul de l'engagement gouvernemental, la loi sur l'ESS n'en atteste pas moins d'un réinvestissement de l'Etat, après un désengagement progressif durant les années 2000. Parallèlement, le contenu de la loi est porteur des valeurs traditionnelles du secteur. Après une vague de modernisation fondée sur une flexibilisation de ces principes pour acclimater des mécanismes capitalistes censés dynamiser le développement des entreprises de l'ESS, l'heure est plutôt à la valorisation des initiatives et à un renforcement des acquis ou des pratiques. Les évolutions de la période antérieure ne sont pas remises en cause, pour partie même prolongées, mais l'essentiel n'est plus là.

(5) Pour une étude technique plus systématique, nous renvoyons à un article à paraître début 2015 dans la Revue des sociétés. Il n'est pas question dans cette rapide présentation d'exposer de façon exhaustive les apports de la loi ESS ${ }^{(5)}$. Notre ambition sera plus synthétique: nous voudrions saisir l'orientation nouvelle. Nous essaierons de montrer comment les deux axes qui nous semblent caractériser l'esprit insufflé par le 
législateur se traduisent. Le soutien des pouvoirs publics se matérialise principalement dans l'élaboration d'un cadre juridique pour le secteur, en ce compris les bases de la détermination de la politique publique le concernant. Quant à la fin de la libéralisation, elle se traduit par diverses innovations, qu'il est difficile de ramasser en une idée unique sans tomber dans l'idéologie ou le parti pris. Elles consistent, d'abord, dans des mesures financières, mais celles-ci attestent déjà que les techniques capitalistes ne sont plus les seules qui retiennent l'attention. La loi approfondit ainsi des mécanismes traditionnels de l'ESS, multiplie les hypothèses de partenariat, tout comme elle tâche d'améliorer la sécurité juridique pour les entreprises du secteur. Nous envisagerons successivement ces différents points, tout en rappelant que nous ne rendrons pas compte de la totalité des dispositions de la loi.

\section{Eléments d'un cadre juridique de l'ESS}

\section{Définition}

Nous laissons de côté la nouvelle définition donnée de la coopérative (loi de 1947, art. 1, al. 1) ${ }^{(6)}$, plus ramassée et plus adaptée à la période. Tout au plus peut-on regretter la référence exclusive à l'intérêt collectif des membres, alors même que l'introduction de la société coopérative d'intérêt collectif (Scic) au sein de la loi générale de 1947 s'était expliquée par le souhait d'en faire autre chose qu'une nouvelle catégorie spéciale. Mais l'essentiel est ailleurs, dans la définition de l'ESS elle-même et de ses entreprises. L'ESS est définie par des principes (art. 1.I) ${ }^{(7)}$ que l'on peut, pour l'un ou l'autre, rattacher à ses diverses familles, mais qui caractérisent certainement de façon synthétique le secteur dans son ensemble: objectif autre que le partage des bénéfices; gouvernance démocratique, transparente et participative; gestion orientée vers le développement de l'activité avec des réserves impartageables. Encore faut-il préciser que l'ESS n'est pas appréhendée seulement dans une vision micro, celle de ses entreprises. Les premiers mots de la loi affirment en effet que l'ESS est un «mode d'entreprendre et de développement économique».

(6) Nous ne mentionnons pas les dispositions modificatives de la loi ESS, mais les dispositions elles-mêmes modifiées afin d'éviter les lourdeurs. (7) Lorsque nous n'indiquons pas la loi dont relèvent les articles que nous citons, c'est qu'il s'agit de la loi ESS elle-même, celle-ci ne comportant pas seulement des dispositions modificatives, mais aussi des dispositions autonomes.

Une importante innovation, très discutée, a été l'abandon de la définition purement statutaire du contour de l'ESS. Les acteurs qui réalisent les activités de production, de transformation, de distribution, d'échange et de consommation de biens et de services sont d'abord, sans doute, les acteurs historiques (art.1.II.1 ${ }^{\circ}$ ), à savoir les coopératives, les mutuelles (au sens large), les associations et les fondations; peut-être faut-il avoir une conception large et inclure les nouveaux fonds de dotation. Mais la définition comprend aussi les sociétés commerciales qui, outre le respect des principes de l'ESS sus-évoqués, poursuivent un objectif d'utilité sociale et prélèvent $20 \%$ des bénéfices de l'exercice pour un fonds de développement, affectent $50 \%$ des bénéfices au report bénéficiaire et aux réserves obligatoires et n’amortissent pas leur capital (art. 1.II. $2^{\circ}$ ). Ces sociétés commerciales ne sont autorisées à faire publiquement état de leur appartenance à l'ESS qu'à la condition d'être immatriculées au registre du commerce et des sociétés (RCS) avec la mention « entreprise de l'ESS » (art. 1.III). Il est difficile d'apprécier l'utilité 
de cette disposition. Elle marque la relative méfiance vis-à-vis de la définition statutaire de l'ESS et, à ce titre, peut se revendiquer du courant de l'économie solidaire ou, plus récemment, de l'entrepreneuriat social. Les revendications portées par ces courants n'ont toutefois pas été suivies jusqu'à leur terme et les critères substantiels ne sont utilisés que pour inclure de nouvelles entreprises dans l'ESS, et non pour en exclure certaines qui remplissent les critères statutaires ${ }^{(8)}$. L'idée d'un label a également été

(8) Il faut relever qu'à notre connaissance seul le Luxembourg s'est orienté dans cette voie, et encore de façon indirecte. abandonnée. La question est donc de savoir si l'ouverture faite aux sociétés commerciales suscitera un grand intérêt. Outre certaines exceptions de taille très réduite, qui ne méritaient pas le coût de la mise en place d'une telle innovation, il est permis de douter du succès: les conditions exigées pour la qualification sont très lourdes (beaucoup plus sévères que certains statuts de l'ESS), et les avantages retirés, malheureusement à la hauteur (réduite) de l'investissement public en faveur de l'ESS. On ne peut que se féliciter de cette innovation sur le plan théorique, que ni la loi espagnole ni la loi québécoise n’ont adoptée, tout en restant sceptique quant à son utilité pratique.

L'article 2 complète l'arsenal par la définition de l'utilité sociale (art. 3). Plus exactement, il détermine les critères à remplir, de façon alternative, pour que les entreprises soient considérées comme poursuivant ce but: elles apportent un soutien à des personnes en situation de fragilité, contribuent à la lutte contre les exclusions et les inégalités ou concourent au développement durable, à la transition énergétique ou à la solidarité internationale, à condition que leur activité soit liée à l'un des deux précédents objectifs. A côté de cette définition formelle, on peut relever que la loi ESS envisage explicitement certaines activités, ce qui signifie qu'elles font partie, dans l'esprit du rédacteur, de l'ESS: il s'agit des éco-organismes (art. 88-92) et du commerce équitable (art. 94). Etant donné que l'appartenance à l'ESS se décide entreprise par entreprise, il est clair cependant qu'il ne s'agit que d'un préjugé sans incidence sur la qualification de celles qui exercent ces activités. La même remarque vaut pour les entreprises d'insertion par l'activité économique (EIAE), qui auront des représentants au sein du Conseil supérieur de l'ESS (art. 4.VI.5 ${ }^{\circ}$ ). Relevons encore que l'entreprise sociale n'est pas totalement oubliée, puisqu'elle constitue l'un des objets de ce dernier (art. 4).

De façon moins emblématique, mais par des dispositions de détail, la construction de l'ESS en un secteur unifié et autonome se poursuit. Ainsi, la dévolution désintéressée de la coopérative ne se fera plus, outre à d'autres coopératives, au bénéfice d'une œuvre d'intérêt général ou professionnel, mais d'une entreprise de l'ESS (loi de 1947, art. 19). De même, la fluidité des organisations du secteur est améliorée: transformation d'un fonds de dotation en fondation (loi $\mathrm{n}^{\circ} 2008-77,6$ art. 140.XI), transformation d'une association en fondation reconnue d'utilité publique (loi n 87-571, art. 20-2), précision des effets de la transformation d'une association en Scic (loi de 1947, art. quindecies et sexdecies), facilitation de la transformation des ONG en Scic par la précision de l'objet de ces dernières (loi de 1947, art. quinquies). On relèvera, seulement à cette occasion, que plusieurs de ces hypothèses concernent la transformation d'une association en une autre organisation. 


\section{Structuration institutionnelle}

\section{StRUCTURATION GLOBALE}

L'article 4 institue un Conseil supérieur de l'ESS chargé d'assurer le dialogue entre les acteurs de l'économie sociale et solidaire et les pouvoirs publics nationaux et européens (al. 1). Celui-ci est consulté pour l'élaboration des règles juridiques du secteur et propose son évaluation (al. 2). Il contribue à l'élaboration d'une stratégie nationale triennale (al. 3) et développe sa propre stratégie de promotion de l'ESS auprès des jeunes (al. 4-7) et en faveur de la parité homme-femme au sein de l'ESS (al. 8-11). Sa composition est classique (VI) et son détail, qui donnera certainement lieu à des luttes assassines dans le secteur, ne sera connu qu'à la publication du décret (VII). Relevons cependant que ce conseil n'est qu'un organe consultatif et qu'il ne s'oriente pas vers un statut d'autorité administrative indépendante. Il est encore intéressant de constater que son domaine d'intervention couvre des questions parfois discutées, comme l'innovation sociale, pour lesquelles il doit définir des orientations permettant d'identifier les projets qui s'y rattachent (art. 15.III).

La Chambre française de l'ESS, définie à l'article 5, assure sur le plan national la représentation et la promotion de l'ESS et des intérêts des entreprises de l'ESS auprès des pouvoirs publics nationaux, sans préjudice des missions des organismes sectoriels. Elle a la forme d'une association dotée des pouvoirs d'une association reconnue d'utilité publique (Arup) et se compose des organisations nationales illustrant les différentes formes statutaires de l'économie sociale et solidaire, y compris les sociétés commerciales, et de représentants du CNcress. Ce dernier devra soutenir, animer et coordonner le réseau des Cress et consolider au niveau national les données économiques et qualitatives recueillies par elles (art. 6, al. 2). La Chambre française a une mission politique, le Conseil national, une mission technique. Aurait-on perdu plus d'énergie à convaincre de l'irrationalité de cette dualité qu'à la laisser perdurer? Les Cress (art. 6) réalisent sur le plan local ce qui n'est pas possible sur le plan national et remplissent les mêmes fonctions que ses deux supérieurs. Elles sont donc une association disposant des mêmes pouvoirs que la Chambre française. Il convient de relever qu'elles tiennent à jour et assurent la publication de la liste des entreprises de l'ESS (al.11) et qu'elles "ont qualité pour ester en justice aux fins, notamment, de faire respecter par les entreprises de leur ressort et relevant du $2^{\circ} \mathrm{du}$ II de l'article $1^{\text {er }}$ de la présente loi l'application effective des conditions fixées à ce même article» (al.10). Cette dernière formulation est malheureuse car, conformément à la vérité, la fonction de la Cress en cette matière ne pourra consister en pratique qu'à solliciter la suppression de la mention ESS du RCS pour les entreprises contrevenantes. La loi s'est préoccupé de la politique publique, mais uniquement sur le plan régional. On peut argumenter que ce niveau est le plus pertinent en termes d'efficacité, mais le constat est peut-être plus prosaïquement l'incapacité de l'Etat à s'impliquer réellement en faveur de l'ESS. Quoi qu'il en soit, la région est en première ligne, en conformité avec l'existant, il faut bien le reconnaître. Elle élabore une stratégie régionale (art.7) en concertation avec la Cress et les entreprises concernées. Elle peut aussi contractualiser avec les organisations territoriales inférieures. L'implication de l'Etat 
se situe également au niveau régional par l'organisation, avec le président du conseil régional, d’une conférence régionale de l'ESS (art. 8, al. 1), au moins biennale, réunissant acteurs de l'ESS, élus territoriaux et partenaires sociaux. Ce temps fort est un élément d'élaboration de la politique publique, qui peut aller plus loin dans la co-construction (art. 8, al. 2-3).

\section{STRUCTURATION SECTORIELLE}

Le Conseil supérieur de la coopération a été créé en 1918, mais est tombé en désuétude, avant d'être ravivé par le décret n 76-356 du 20 avril 1976. Il se trouve aujourd'hui conforté par l'onction législative (loi de 1947, art. 5-1). Ses fonctions sont celles des organes consultatifs dans son secteur, avec une composition précisée par décret. Outre son intervention déjà prévue dans la procédure de sortie du statut coopératif, il dispose d'une fonction en matière de définition des modalités de la révision coopérative (art. 5-1, al. 5). La question est de savoir si l'intervention législative donnera à l'organe une vitalité qui lui faisait défaut. Ce ne sera pas le cas si son apathie est inversement proportionnelle à la conscience collective du secteur.

Dans un même mouvement, l'article 63 de la loi ESS consolide le Haut Conseil à la vie associative (HCVA). Il s'agit de consacrer dans la loi une instance qui existe depuis plusieurs décennies sous la forme du Conseil national de la vie associative (CNVA), avant d'être réformé et renommé Haut Conseil à la vie associative par le décret $n^{\circ} 2011-773$ du 28 juin 2011. Celui-ci remplit le rôle classique de consultation et de proposition règlementaire et de politique publique sur la vie associative. Tout au plus peut-on relever un mode original de saisine du Haut Conseil sur un point déterminé, à l'initiative d'" au moins cent associations couvrant au moins trois régions et ayant un objet statutaire comparable».

\section{Mesures financières}

\section{Titres financiers}

Sans qu'il s'agisse d'un titre financier dans son sens habituel, il faut noter ici que les monnaies locales complémentaires fleurissant dans divers territoires en dehors de tout cadre juridique sont prévues par la nouvelle loi (C. mon. fin., art. L311-5 et s.). Leur émission et leur gestion sont réservées aux entreprises d'ES dont c'est l'unique objet social. C'est peut-être aussi symboliquement le signe d'une autre façon d'appréhender les questions financières.

Au titre des outils financiers de capitalisation, trois innovations ayant en commun de prolonger le mouvement des années 80-90 qui avait multiplié les instruments financiers à disposition des entreprises de l'ESS retiennent l'attention. D'abord, dans le domaine mutualiste, les certificats mutualistes (C. assu., art. L322-26-8 et s.; C. mut., art. L221-19 et s.), auxquels font pendant les certificats paritaires (C. sécu., art. L931-15-1 et s.). Il ne s'agit pas d'une simple transposition des certificats coopératifs créés par la loi de 1992, dans la mesure où les certificats mutualistes ne peuvent pas représenter une partie du capital social, les mutuelles en étant dépourvues. Ils peuvent être souscrits par les adhérents, les assurés des entreprises du groupe, ainsi que par d'autres mutuelles (art. L223-26-8.I). Ils ne sont pas cessibles comme leurs devanciers, 
la cession ne pouvant intervenir que sous forme de rachat par l'émetteur, qui pourra les céder à nouveau dans un délai de deux ans (art. L322-26-9). Ils ont vocation à alimenter les fonds d'établissement. Leur rémunération, fixée annuellement par l'assemblée générale, est variable: un décret fixera la part maximale des résultats du dernier exercice clos et des précédents exercices susceptible d'y être affectée chaque année (art. L322-26-8.V). Le texte précise les informations fournies aux souscripteurs (art. L223-26-8.III) et un décret précisera l'implication de l'Autorité de contrôle prudentiel (art. L223-26-8, al. 9).

En second lieu, les fondations se voient ouverte l'utilisation des titres associatifs (C. mon. fin., art. L213-20-1a). Quant à ces derniers, ils connaissent quelques aménagements dans le but d'accroître leur usage. Il est désormais possible d'émettre des obligations remboursables à une échéance conditionnée à la constitution, depuis la date de l'émission, d'excédents dépassant le montant nominal de l'émission, nets des éventuels déficits constitués durant la même période (C. mon. fin., art. L213-9). Par ailleurs, l'interdiction, directe ou indirecte, de la détention des obligations émises par les associations par les dirigeants de fait ou de droit de l'association est fulminée afin d'éviter toute distribution indirecte des bénéfices (C. mon. fin., art. L213-14).

\section{Facilitation du soutien public et privé}

Un certain nombre de dispositions ont pour objet de faciliter la réunion de fonds au profit des entreprises de l'ESS, mais les mesures ne sont souvent pas exclusivement financières. Outre le suivi statistique du financement de ces entreprises (art.12) et la participation des organismes représentatifs du secteur aux côtés de la Banque publique d'investissement (BPI) pour le suivi de leur accès au financement (art. 17), le soutien public prend différentes formes. On en trouve une trace dans le schéma de promotion des achats publics socialement responsables (art. 13), tout comme dans l'adaptation du Code monétaire et financier aux fonds européens d'entrepreneuriat social (C. mon. fin., art. L214-153-1) ou la consolidation des dispositifs locaux d'accompagnement (art. 61). Les autres mesures n'engagent pas davantage l'Etat. De façon emblématique, une définition de la subvention est introduite dans la loi 2000-321 (article 9-1) relative aux droits des citoyens dans leurs relations avec les administrations, en ouverture de la section sur la transparence financière: "Constituent des subventions, au sens de la présente loi, les contributions facultatives de toute nature, valorisées dans l'acte d'attribution, décidées par les autorités administratives et les organismes chargés de la gestion d'un service public industriel et commercial, justifiées par un intérêt général et destinées à la réalisation d'une action ou d'un projet d'investissement, à la contribution au développement d'activités ou au financement global de l'activité de l'organisme de droit privé bénéficiaire. Ces actions, ces projets ou ces activités sont initiés, définis et mis en œuvre par les organismes de droit privé bénéficiaires. Ces contributions ne peuvent constituer la rémunération de prestations individualisées répondant aux besoins des autorités ou des organismes qui les accordent. " Nous reproduisons cette disposition in extenso, tant elle est jugée propre à assainir la pratique de la subvention et à déjouer 
la perfidie bruxelloise et la crainte révérentielle des collectivités territoriales. A dire vrai, nous ne voyons pas bien en quoi une définition juridique ferait barrage à une évolution pluridécennale des politiques publiques, ni ne couvrirait d'un voile protecteur contre le droit européen de la concurrence. Il est fort à craindre que les vertus incantatoires du langage juridique ne marquent ici leurs limites.

On peut encore relever, au titre de la participation publique, les fonds territoriaux de développement associatif (art. 68). Leurs contours manquent de netteté: il est simplement indiqué que les associations y contribuent, ce qui suppose que ces fonds ne sont pas abondés exclusivement par elles. Une confirmation peut être trouvée auprès des fonds de développement coopératif (art. 23), pour lesquels il est précisé a contrario qu'ils sont financés par les coopératives. Peuvent également être créés des fonds de garantie des apports en fonds associatifs (art. 77), destinés à garantir au bénéfice des associations qui les financent la reprise de ces apports. Plus positivement, le soutien financier des personnes publiques, principalement les collectivités territoriales, aux Scic est rendu plus facile, puisque le plafond de leur participation au capital passe de 20 à $50 \%$ (loi de 1947, art. 19 septies). Finalement, la mobilisation de l'épargne salariale se trouve touchée par la substitution aux entreprises solidaires des entreprises solidaires d'utilité sociale (C. trav., art. L3332-17-1). L’agrément en cette qualité est facilité pour certaines entreprises, notamment dans le secteur de l'insertion.

En marge du soutien financier, le développement de l'ESS est souhaité afin de réaliser la mutation générationnelle des entreprises pour lesquelles tous les indicateurs prédisent des difficultés. C’est le sens des mécanismes destinés à faciliter la reprise des entreprises par leurs salariés. En amont, intervient le dispositif d'information de ces derniers sur leurs possibilités de reprise d'une entreprise, qu'il s'agisse d'un fonds de commerce (C. com., art. L141-23 et s.) ou d'une société (C. com., art. L23-10-1 et s.). En aval, la loi met en place un dispositif d'amorçage pour les Scop (diverses modifications de la loi de 1978 prévues par l'article 27 de la loi ESS). Nous ne développons pas le mécanisme, mais il consiste en un assouplissement des conditions de la transformation d'une société commerciale en Scop. Si l'on souhaite plein succès à ces initiatives, on attendra quelques années pour en évaluer l'importance quantitative.

\section{Approfondissement des mécanismes de $1^{1}$ ESS}

Si déjà les aspects financiers offraient une coloration toute différente de celle des grandes réformes des années $80-90$, la nouvelle orientation est encore plus perceptible par la recherche d'une plus grande effectivité des principes traditionnels de l'ESS. La mesure phare dans cette direction consiste certainement dans le guide définissant les conditions d'amélioration continue des bonnes pratiques des entreprises de l'ESS (art. 3) que doit réaliser le Conseil supérieur de l'ESS dans l'année qui suivra la publication du décret précisant sa composition et son fonctionnement. Etant donné l'étendue de son contenu et l'obligation de l'adapter aux diverses formes juridiques d'entreprises, nul doute que cette première année d'existence 
sera bien chargée. L'utilisation concrète de ce guide n'est pas claire: il est avant tout conçu comme une orientation destinée aux assemblées générales des entreprises, mais aussi à leurs salariés, le tout sous le regard du Conseil supérieur de l'ESS.

On peut relever d'autres mesures qui attestent de cette volonté d'approfondissement des principes de l'ESS - nous le ferons sans les détailler, faute de place. Il s'agit d'abord du renforcement de la démocratie au sein des sociétés d'assurance mutuelle (C. assu., art. L322-26-1-1, L322-26-2). Un rapport devra en outre être rendu pour déterminer s'il convient de renforcer les droits des administrateurs de mutuelle (art. 52) et s'il faudrait transposer aux sociétés d'assurance mutuelle le dispositif quant à l'implication des salariés du secteur privé ou des agents publics dans les mutuelles (art. 58).

Du côté des associations, une place non négligeable est faite au bénévolat. C’est le cas avec la résurrection du volontariat associatif (art. 64, qui modifie différentes dispositions du Code du service national). De façon plus prospective, un rapport sur les congés existant pour favoriser le bénévolat associatif et sur la création d'un congé d'engagement pour l'exercice de responsabilités associatives bénévoles devra être remis dans les six mois (art. 67).

L'évolution est aussi patente, sinon plus, dans le domaine coopératif. Symboliquement, plusieurs principes coopératifs sont déplacés au sein de la loi de 1947 pour intégrer son article $1^{\mathrm{er}}$. D’autres principes implicites sont réaffirmés, tels que la gratuité des fonctions de membre de conseil d'administration ou de surveillance (loi de 1947, art. 6). De façon plus surprenante, mais avec notre entière approbation, les conditions de la sortie du statut coopératif, admise en 1992, sont restreintes (loi de 1947, art. 25): disparition de la possibilité de transformer la coopérative lorsque les nécessités de son développement l'exigent. Mais l'innovation la plus importante dans ce secteur consiste de toute évidence dans la réforme et la généralisation du mécanisme de la révision coopérative (loi de 1947, art. 25-1 et s.). Son objectif a été clarifié: il consiste à vérifier la conformité de leur organisation et de leur fonctionnement aux principes et aux règles de la coopération et à l'intérêt des adhérents, ainsi qu'aux règles coopératives spécifiques qui leur sont applicables et, le cas échéant, à leur proposer des mesures correctives (loi de 1947, art. 25-1), disposition qui existait déjà quasiment à l'identique pour les coopératives agricoles. Tandis que la révision se confondait parfois avec une forme d'audit financier, elle porte aujourd'hui clairement sur le fonctionnement de la coopérative et l'évaluation de sa conformité avec les principes coopératifs. En outre, et ça n’a pas été sans débats, la révision s'applique désormais à toutes les coopératives, notamment bancaires.

\section{Facilitation des partenariats}

\section{Les restructurations}

Toutes les entreprises de l'ESS sont concernées par le mouvement de concentration, alors que les modalités juridiques ne sont toujours pas fixées. Celles-ci s'appliquaient aux coopératives par ricochet du droit des sociétés, elles étaient prévues pour les mutuelles, elles le sont aujourd'hui également pour les associations (loi de 1901, art. 9 bis) et les fondations 
(loi $\mathrm{n}^{\circ}$ 87-571, art. 20-1). Sont ainsi réglementés les fusions, les scissions et les apports partiels d'actif, sur le modèle du droit des sociétés, moyennant les adaptations liées à l'organisation particulière de l'association et de la fondation. L'organe compétent pour décider de l'opération est l'assemblée générale de l'association statuant aux conditions requises pour la modification des statuts (loi de 1901, art. 9 bis, al. 1 et 2). Un projet de l'opération, qui est publié dans un journal d’annonces légales, doit être établi (al. 4). Celle-ci emporte transmission universelle du patrimoine et n'implique donc aucune liquidation (al. 6). La situation des créanciers obligataires est traitée par renvoi au Code de commerce (al. 8). Afin de sécuriser la situation des associations disposant d'un agrément administratif, à l'exclusion de la reconnaissance d'utilité publique, l'association qui projette de procéder à une opération de restructuration peut interroger l'auteur de l'agrément, qui doit lui répondre (IV). Le régime juridique est identique pour les fondations.

\section{Les groupes}

La nouvelle loi innove également par la diversification des modes d'organisation de groupes au sein de l'ESS ou par la modification des règles préexistantes. Les unions d'entreprises de l'ESS, qui avaient été introduites par voie d'amendement, n'ont finalement pas été retenues, mais un rapport doit être remis au parlement par le gouvernement avant la fin de l'année pour rendre compte de sa faisabilité, par insertion au sein de la loi de 1947 (art. 26).

Plus modestement, l'objet des unions de coopératives est étendu pour comprendre le développement de l'activité de ses membres (loi de 1947, art. 5). La reconnaissance du fonctionnement intégré des réseaux coopératifs a aussi conduit à autoriser au profit des coopératives de commerçants les clauses statutaires de préemption des fonds de commerce exploitées par leurs membres (C. com., art. L124-4-1). De même, les coopératives artisanales "peuvent mettre en œuvre, par tous moyens, une politique commerciale commune, notamment par la réalisation d'opérations commerciales ou publicitaires, pouvant comporter des prix communs » (loi de 1983, art. 1, al. 2). La création d'un groupement de Scop est plus ambitieuse (loi de 1978, art. 47 bis et s.). Cette structure implique une grande proximité entre les coopératives participantes, dans la mesure où certaines de leurs stipulations statutaires doivent être identiques (art. 47 bis, al.3) et ne pourront plus être modifiées individuellement par une coopérative (art. 47 ter, al. 3). Certaines décisions doivent aussi être adoptées dans les mêmes termes, comme l'acceptation d'un nouveau membre (art. 47 ter, al. 2) et la transformation d'une partie des excédents nets en parts sociales (art. 47 bis, al. 9). L'intérêt de constituer un tel groupement réside d'abord dans le fait que les salariés employés par l'une des sociétés membres du groupement sont assimilés à des coopératives pour le calcul des limitations de droits de vote, en application de l'article 3 bis de la loi de 1947 au sein des autres coopératives du groupement. Plus encore, il est même possible à une Scop de détenir 51 \% des parts d'une autre Scop du groupement, à la condition que les salariés des Scop filiales détiennent un pourcentage minimal de capital de la Scop mère (art. 47 quinquies). La même solution vaut pour une filiale capitaliste que la Scop mère déciderait de transformer en Scop sans égard au délai de dix ans 
normalement applicable (art. 47 sexies). L'initiative est intéressante, surtout si elle conduit les grosses Scop à coopérativiser les filiales qu'elles ont constituées pour leur développement. Pour le reste, la volonté des Scop de s'engager dans de tels groupements intégrés reste à démontrer.

Finalement, une nouvelle union a été introduite dans le Code de la mutualité (C. mut., art. L111-4-3) entre mutuelles et unions pratiquant la prévention, l'action sociale et la gestion de réalisations sanitaires et sociales ou entre des mutuelles et des unions pratiquant des opérations d'assurance, de réassurance et de capitalisation. Elles ont pour objet de faciliter et de développer, en les coordonnant, des activités sanitaires, sociales et culturelles. Ces unions peuvent accueillir comme membres les entreprises statutaires de l'ESS (al. 2-8), mais les mutuelles du Code de la mutualité ont la majorité absolue à l'assemblée comme dans l'organe d'administration (al.13). Ses statuts peuvent lui conférer un pouvoir de contrôle financier sur les activités sanitaires, sociales et culturelles de ses membres (al. 14).

\section{Les partenariats}

Tout d'abord, la loi ESS encadre les contrats de co-assurance (C. sécu., art. L932-13-2 et L932-14-1; C. mut., art. L227-1 et s. ; C. assu., art. L145-1 et s.); nous nous contentons de le mentionner. De façon plus globale à l'ESS, l'article 9 définit les pôles territoriaux de coopération économique (PTCE). Ceux-ci sont constitués par un regroupement sur un même territoire d'entreprises de l'ESS, d'entreprises capitalistes, de collectivités territoriales, d'organismes de recherche ou de formation et de toute personne physique ou morale pour mettre en œuvre une stratégie commune et continue de mutualisation, de coopération ou de partenariat au service de projets économiques et sociaux innovants, socialement ou technologiquement, et porteurs d'un développement local durable. La définition recouvre les multiples expériences en cours. Il est seulement précisé que la sélection des PTCE soutenus par l'Etat se fait par un comité interministériel associant les financeurs, après avis de personnalités qualifiées et de représentants de collectivités territoriales. Les PTCE sont à la mode et leur sécurisation législative est bienvenue.

\section{Amélioration de la sécurité juridique}

\section{Consécration de mécanismes juridiques préexistants}

Sans insister, il nous semble important de mentionner la consécration par la loi ESS de la coopérative d'activités et d'emploi (CAE). Cette dernière est intégrée à la loi de 1947 en un titre III ter contenant un unique article 26-41. Cette place est un peu étrange. En dépit de toute l'admiration que l'on peut nourrir pour ces nouvelles coopératives, on ne voit pas bien pourquoi elles se retrouvent dans la loi portant statut général de la coopération. La volonté de ne pas les inclure dans la loi de 1978 sur les Scop ne saurait être une justification suffisante. Qui plus est, la définition donnée de ces coopératives est décevante: elles "ont pour objet principal l'appui à la création et au développement d'activités économiques par des entrepreneurs personnes physiques ». Cette notion d'appui, qui fait pendant au contrat d'appui au 
projet d'entreprise, est réductrice. La loi est globalement plus satisfaisante dans la détermination du statut de l'entrepreneur salarié et de ses relations avec la coopérative, incluse dans un nouveau titre du Code du travail consacré aux entrepreneurs salariés associés d'une CAE (C. trav., art. L7331-1 et s.). La protection du statut de salarié est confirmée, ce qui est l'essentiel. Le reste requerrait une analyse détaillée qui ne trouve pas sa place ici.

Finalement, hors de la loi ESS, il nous semble utile de relever deux initiatives de 2014 qui approfondissent un concept coopératif central: la double qualité. C'est le cas à travers le contrat coopératif des coopératives d'habitants (C. const. hab., art. L201-8) comme du lien entre le coopérateur et la coopérative agricole (C. rur., art. L521-1-1).

\section{Extension de la liberté des acteurs de l'ESS}

L'extension de la liberté des acteurs de l'ESS se manifeste soit lors de l'élaboration des statuts, soit lors de l'exécution des actes du groupement. $\mathrm{Au}$ stade de l'élaboration des statuts, plusieurs coopératives voient leur possibilité de choix de forme sociale étendue. C'est le cas pour la Scop (loi de 1978, art. 3) ${ }^{(9)}$ et la Scic (loi de 1947, art. 19 quinquies), qui peuvent désormais adopter la forme de société par actions simplifiée (SAS). Dans le

(9) Bertrel M., 2014, « La Scop SAS : une nouvelle opportunité en faveur de l'entrepreneuriat ", Recma, $\mathrm{n}^{\circ} 332$, avril, p. 124 même esprit, les coopératives de commerçants ne sont plus tenues d'adopter la forme de société anonyme, mais peuvent aussi revêtir celle de société à responsabilité limitée (SARL; C. com., art. L124-3). Cette flexibilité est parfaitement conforme à l'esprit de liberté et d'imagination qui anime le mouvement ESS. On peut rattacher à la même veine l'abandon du strict exclusivisme dans les coopératives de commerçants (C. com., art. L124-2), puisqu'elles sont soumises au nouveau droit commun en la matière, qui autorise les transactions avec les tiers à hauteur de $20 \%$ (loi de 1947, art. 3).

Au stade de l'accomplissement des actes juridiques par l'entreprise, la capacité juridique des associations est renforcée. La capacité juridique spéciale des associations d'assistance, de bienfaisance, de recherche scientifique ou médicale est supprimée. A la place, une capacité juridique accrue est généralisée à toutes les associations dites d'intérêt général, en d'autres termes celles dont les donateurs bénéficient de la réduction d'impôts de 66 \% (CGI, art. 200.1.b) ${ }^{(10)}$. La capacité accrue comporte deux éléments: d'une part, la

(10) La catégorie n'est pas générale, mais correspond à une liste figurant dans cette disposition du Code général des impôts. Elle est beaucoup plus large que celle antérieurement visée à l'article 6 de la loi de 1901. capacité de recevoir des dons et des legs (avec le mécanisme de la déclaration préalable mis en place en 2005) et, d'autre part, posséder et administrer tous immeubles acquis à titre gratuit. Cette extension de capacité juridique est toutefois subordonnée à l'écoulement d'un délai de trois ans depuis leur déclaration. Cette évolution, profitable aux associations, est simplificatrice, puisqu'elle coordonne des catégories juridiques autrefois distinctes et ne peut donc qu'être approuvée. La même remarque vaut pour les Arup (loi de 1901, art.11): d'une part, leur aptitude à être propriétaire d'immeuble ne connaît plus de restriction; d'autre part, la règlementation de leurs placements financiers est révisée pour être calquée sur celle des institutions et des unions exerçant une activité d'assurance, tels qu'ils sont régis par le Code de la Sécurité sociale. Finalement, le gouvernement est autorisé 
à légiférer par ordonnance " afin de simplifier les démarches des associations et des fondations auprès des administrations, notamment en adaptant les modalités d'enregistrement, d'agrément et de reconnaissance d'utilité publique et les conditions d'obtention de financements» (art. 62).

Plusieurs conclusions peuvent être tirées de cette rapide revue. Tout d'abord, la loi a totalement validé le concept d'économie sociale et solidaire et opéré un mariage réussi, si l'on se réfère aux inspirations variées qui parviennent à un produit substantiellement cohérent. Ensuite, l'entrepreneuriat social et l'innovation sociale ne font qu'une timide entrée dans le droit positif français, ce qui confirmerait l'hypothèse qu'il s'agit d'un concept européen fédérateur n'ayant pas vocation à se substituer aux conceptions nationales. Finalement, le chemin est encore long pour parvenir à un droit de l'économie sociale et solidaire. Pourtant, le besoin n'en est pas totalement illusoire, il suffit de relever les dispositions répétées dans plusieurs codes afin de couvrir l'ensemble des lois spéciales.

Est-ce une loi importante? Sans conteste. Elle ne bouleversera certainement pas les pratiques, et on a même vu la fragilité de l'impulsion politique qu'elle est censée représenter. Il n'empêche qu'elle cristallise les changements de perspective antérieurs à la crise à l'intérieur de l'ESS, diffusés à l'extérieur avec elle. Certaines dispositions techniques sont importantes. Elles sont en tout cas nombreuses et toucheront en conséquence toutes les entreprises de l'ESS. Mais nous vivons de symboles et l'essentiel demeure dans l'institutionnalisation du secteur, avec la mise en place de ses organes et la prévision de ses grand-messes. Il y aurait de nombreuses raisons d'être chagrin, tant pour des considérations techniques que politiques, mais regardons un peu en arrière, et le chemin parcouru ne semblera plus négligeable. Le reste, comme toujours, c'est aux acteurs de le construire. 